\title{
Квазиклассическая модель статической электропроводности сильно легированных вырожденных полупроводников при низких температурах
}

\author{
(C) Н.А. Поклонский, С.А. Вырко, А.Н. Деревяго \\ Белорусский государственный университет, \\ 220030 Минск, Республика Беларусь \\ E-mail: poklonski@bsu.by
}

(Получена 17 мая 2017 г. Принята к печати 2 июня 2017 г.)

\begin{abstract}
Рассматриваются кристаллы германия, кремния, арсенида галлия и антимонида индия $n$-типа на металлической стороне перехода изолятор-металл (перехода Мотта). В квазиклассическом приближении рассчитываются статическая (на постоянном токе) электрическая проводимость и дрейфовая подвижность электронов $c$-зоны, а также электростатические флуктуации их потенциальной энергии и порог подвижности. Считается, что единичный акт упругого кулоновского рассеяния подвижного электрона происходит только в сферической области кристаллической матрицы, в центре которой расположен ион примеси. Результаты расчетов по предложенным формулам без использования подгоночных параметров численно согласуются с экспериментальными данными в широком диапазоне концентраций водородоподобных доноров при слабой и умеренной их компенсации акцепторами.
\end{abstract}

DOI: 10.21883/FTP.2018.06.45913.8651

\section{1. Введение}

Задача количественного описания миграции электронов и дырок в трехмерных кристаллических полупроводниках при увеличении в них концентрации атомов примесей и достижении ими квазиметаллического состояния по-прежнему важна для приложений (см., например, [1-3]). Сильно легированные вырожденные полупроводниковые материалы $n$ - и $p$-типа электропроводности являются радиационно стойкими элементами приборов криогенной микро- и оптоэлектроники [4-6]. Их электрические и оптические параметры слабо зависят от флюенса радиации даже при низких температурах, что важно для работы приборов в космосе (температура реликтового излучения $\approx 2.7 \mathrm{~K}$ ). Для целей силовой электроники актуально аналитическое описание электропроводности полупроводниковых элементов в размыкателях тока $[7,8]$. Одной из практически важных задач является создание трехмерных электродов к двумерному проводнику электричества (например, к графену) с согласованными значениями их сопротивлений в электрической цепи.

Отметим, что, хотя первые эксперименты по измерению электрического сопротивления в контролируемо сильно легированных вырожденных полупроводниках при температурах жидкого гелия и азота были выполнены полвека назад (см., например, [9-12]), до сих пор нет теории, количественно объясняющей накопленные данные опытов. Например, в работе [1] предложен способ аналитической аппроксимации подвижности основных носителей заряда в кристаллических полупроводниках.

Величина электрической проводимости определяется степенью электрической активности неподвижных в кристаллической матрице атомов примесей (см., напри- мер, [13]). Обычно при сильном легировании моноатомных полупроводников (например, $\mathrm{Ge}, \mathrm{Si}$ ) происходит некоторое уменьшение электрической активности основной (легирующей) примеси вследствие перехода ее части из узлов в междоузлия или образования энергетически неактивных ассоциатов из примеси. В бинарных кристаллических полупроводниковых соединениях (например, GaAs и InSb) атомы легирующей примеси могут частично размещаться в двух подрешетках, что приводит к уменьшению ее электрической активности. В основном электроны и дырки в сильно легированных полупроводниках при криогенных температурах рассеиваются на ионах примесей.

Цель работы - расчет электропроводности на постоянном токе сильно легированных вырожденных кристаллических полупроводников $n$-типа при учете упругого кулоновского рассеяния подвижных электронов на ионах водородоподобных примесей при низкой температуре. Для определенности рассматривается кристаллический полупроводник $n$-типа, так как дрейфовая подвижность электронов выше, чем дырок. Вследствие этого относительный вклад рассеяния на ионах примесей в уменьшение подвижности электронов больше, чем в уменьшение подвижности дырок.

В сильно легированном полупроводнике $n$-типа все доноры со средней концентрацией $N$ находятся в зарядовом состоянии $(+1)$, а все акцепторы со средней концентрацией $K N$ (где $0<K<1-$ степень компенсации) в зарядовом состоянии $(-1)$. Из условия электрической нейтральности следует, что средняя концентрация всех электронов в $c$-зоне $n=(1-K) N$ меньше концентрации ионов $N_{\text {ion }}=(1+K) N$. При низких температурах переходы электронов из $v$-зоны в $c$-зону несущественны для поддержания ионизационного равновесия. 
Критерием вырожденного полупроводника $n$-типа при абсолютной температуре $T \rightarrow 0$ будем считать концентрацию электронов, соответствующую металлической стороне перехода Мотта $[14,15]$. Переход из изоляторного состояния в металлическое при $T \rightarrow 0$ (переход Мотта) $[16,17]$ происходит, когда $n>n_{\mathrm{M}}$, где $n_{\mathrm{M}}$ - критическая концентрация подвижных электронов $c$-зоны, соответствующая переходу Мотта. Величина $n_{\mathrm{M}}=(1-K) N_{\mathrm{M}}$ (в пределе нулевой температуры $T \rightarrow 0)$ определяется, согласно [15], соотношением

$$
\frac{N_{\mathrm{M}}^{1 / 3} a_{\mathrm{B}}}{v^{2 / 3}}=\frac{0.1}{(1-K)^{2 / 3}},
$$

где $a_{\mathrm{B}}=4 \pi \varepsilon_{r} \varepsilon_{0} \hbar^{2} / m_{n d}^{(1)} e^{2}-$ боровский радиус для электрона $c$-зоны в одной из $v$ эквивалентных долин $(v=6$ для $n$-Si, $v=4$ для $n$-Ge, $v=1$ для $n$-GaAs и $n$-InSb) с эффективной массой $m_{n d}^{(1)} ; \varepsilon=\varepsilon_{r} \varepsilon_{0}-$ абсолютная статическая диэлектрическая проницаемость полупроводника, $\varepsilon_{r}$ - относительная проницаемость, обусловленная электронами $v$-зоны на фоне нейтрализующих их положительно заряженных остовов кристаллической матрицы, $\varepsilon_{0}-$ электрическая постоянная, $e-$ элементарный заряд; $N_{\mathrm{M}}^{1 / 3} a_{\mathrm{B}}$ - параметр Мотта. Из формулы (1) видно, что чем меньше число долин $v$ в $c$-зоне и меньше степень компенсации $K$ полупроводника, тем меньше концентрация основных примесей $N_{\mathrm{M}}=n_{\mathrm{M}} /(1-K)$, необходимая для реализации перехода Мотта.

В данной работе квазиклассическая теория упругого рассеяния электронов $c$-зоны на ионах водородоподобных примесей, предложенная в работах $[18,19]$ для слабо легированных полупроводников, применяется к сильно легированным вырожденным полупроводникам $n$-типа. Далее считается, что сферическая область кристаллической матрицы, в которой происходит один акт рассеяния электрона в кулоновском поле одного иона примеси, имеет объем $4 \pi R_{\text {ion }}^{3} / 3=N_{\text {ion }}^{-1}$, где $N_{\text {ion }}=(1+K) N-$ средняя концентрация ионов примесей. Диаметр этой области

$$
2 R_{\text {ion }}=1.24 N_{\text {ion }}^{-1 / 3}
$$

приблизительно равен среднему расстоянию между ионами $\left(\approx 1.28 N_{\text {ion }}^{-1 / 3}\right)$, определенному в [20] методом полиэдров Вороного-Дирихле. Длительность одного акта электрон-ионного взаимодействия (рассеяния) равна времени пролета электроном $c$-зоны сферической области, приходящейся на один ион примеси в кристалле. При этом используется борновское приближение и волновая функция электрона в виде плоской волны (приближение эффективной массы).

Далее рассматриваются два вида сильно легированных вырожденных кристаллических полупроводников $n$-типа: условно идеально легированные [символ (id)] и реально легированные [символ (re)], которые различаются только зависимостью одноэлектронной энергии краев зон разрешенных энергий $E_{c}$ и $E_{v}$ от пространственных координат $x, y, z$. Для id-кристаллов $E_{c}$ и $E_{v}$ не зависят от $x, y, z$, т. е. дно $c$-зоны и потолок $v$-зоны плоские. Для ге-кристаллов $E_{c}(x, y, z)$ и $E_{v}(x, y, z)$ являются антисимметричными функциями относительно середины запрещенной энергетической зоны [16,21] из-за электростатических флуктуаций потенциальной энергии и электронов $c$-зоны $W_{n}$ и дырок $v$-зоны $W_{p}$ (рис. 1$)$. Средние концентрации электронов $n^{(\mathrm{id})}=n^{(\text {re) }}=n$, доноров $N$ и акцепторов $K N$ для id- и re-полупроводников считаются равными.

\section{2. Статистика электронов в идеальных и реальных кристаллических полупроводниках при низких температурах}

$\mathrm{B}$ id-полупроводнике $n$-типа (с плоскими краями зон разрешенных значений одноэлектронной энергии $E_{n}$, т. е. когда $W_{n}=W_{p}=0$ и $E_{\mathrm{mob}}^{(c)}=E_{c}=0$; рис. 1) концентрация электронов в $c$-зоне $n^{(\mathrm{id})}$ при условии полной ионизации доноров с концентрацией $N$ и акцепторов с

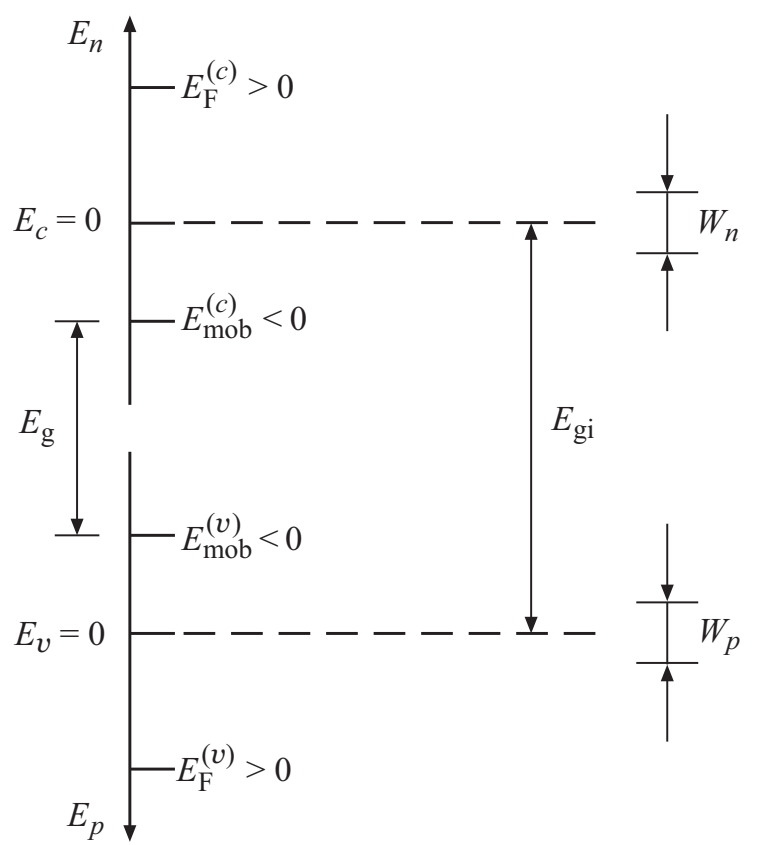

Рис. 1. Схема отсчета уровней энергии электронов и дырок в сильно легированных вырожденных кристаллических полупроводниках $n$-типа (верхняя шкала) и $p$-типа (нижняя шкала): $E_{c}-E_{v}=E_{\mathrm{gi}}-$ ширина запрещенной энергетической зоны (щели) нелегированного (собственного) кристалла, $E_{\mathrm{g}}=E_{\mathrm{gi}}-\left|E_{\text {mob }}^{(c)}\right|-\left|E_{\mathrm{mob}}^{(v)}\right|-$ ширина энергетической щели легированного кристалла, $E_{n}-$ энергия электрона $c$-зоны, $E_{\mathrm{F}}^{(c)}>0-$ уровень Ферми для электронов в полупроводнике $n$-типа, $E_{\mathrm{mob}}^{(c)}<0-$ порог подвижности для электронов; $E_{p}-$ энергия дырки $v$-зоны, $E_{\mathrm{F}}^{(v)}>0-$ уровень Ферми для дырок в полупроводнике $p$-типа, $E_{\mathrm{mob}}^{(v)}<0-$ порог подвижности для дырок; $W_{n}, W_{p}$ - среднеквадратичные флуктуации потенциальной энергии электронов $(n)$ и дырок $(p)$. 
концентрацией $K N$ равна (см., например, $[16,22])$ :

$$
n^{(\mathrm{id})}=\frac{1}{V} \int_{E_{c}=0}^{\infty} g_{n}^{(\mathrm{id})}\left(\varepsilon_{\mathrm{kin}}\right) f_{n}\left(\varepsilon_{\mathrm{kin}}\right) d \varepsilon_{\mathrm{kin}}=(1-K) N,
$$

где $V-$ объем трехмерного кристаллического образца, $g_{n}^{\text {(id) }}\left(\varepsilon_{\text {kin }}\right)=V\left[\left(2 m_{n d}\right)^{3 / 2} / 2 \pi^{2} \hbar^{3}\right] \varepsilon_{\text {kin }}^{1 / 2}-$ плотность одноэлектронных состояний в $c$-зоне кристалла, $f_{n}\left(\varepsilon_{\text {kin }}\right)=\left\{1+\exp \left[\left(\varepsilon_{\text {kin }}-\varepsilon_{\mathrm{F}}^{(c)}\right) / k_{\mathrm{B}} T\right]\right\}^{-1}-$ функция Ферми-Дирака, $\varepsilon_{\mathrm{kin}}-$ кинетическая энергия электрона $c$-зоны, $\varepsilon_{\mathrm{F}}^{(c)}-$ химический потенциал, $k_{\mathrm{B}}-$ постоянная Больцмана, $T$ - абсолютная температура; $m_{n d}=v^{2 / 3} m_{n d}^{(1)}-$ эффективная масса плотности состояний электронов в $c$-зоне, $v-$ число долин (эквивалентных минимумов энергии электронов в $c$-зоне), $m_{n d}^{(1)}-$ эффективная масса плотности состояний электронов в одной долине, $\hbar=h / 2 \pi-$ постоянная Планка.

Из (3), когда химический потенциал $\varepsilon_{\mathrm{F}}^{(c)}$ более чем в 3 раза превышает тепловую энергию $k_{\mathrm{B}} T$ и ферми-газ электронов в $c$-зоне id-полупроводника можно считать вырожденным, получаем (см., например, $[16,17,22])$

$$
\varepsilon_{\mathrm{F}}^{(c)}=\xi_{\mathrm{F}}^{(c)}\left[1-\left(\pi^{2} / 12\right)\left(k_{\mathrm{B}} T / \xi_{\mathrm{F}}^{(c)}\right)^{2}\right]>3 k_{\mathrm{B}} T,
$$

где $\xi_{\mathrm{F}}^{(c)}=\left(\hbar^{2} / 2 m_{n d}\right)\left[3 \pi^{2} n^{(\mathrm{id})}\right]^{2 / 3}-$ энергия Ферми, т.е. химический потенциал $\varepsilon_{\mathrm{F}}^{(c)}$ в пределе нулевой абсолютной температуры $(T \rightarrow 0)$.

В трехмерном кристаллическом re-полупроводнике $n$-типа $\left(W_{n} \neq 0\right.$ и $E_{\text {mob }}^{(c)} \neq E_{c}=0$; рис. 1$)$ плотность одноэлектронных состояний в $c$-зоне есть $[23,24]$

$$
g_{n}^{(\mathrm{re})}\left(E_{n}\right)=V \frac{\left(2 m_{n d}\right)^{3 / 2}}{2 \pi^{2} \hbar^{3}} \int_{-\infty}^{E_{n}}\left(E_{n}-U_{n}\right)^{1 / 2} \mathscr{P}\left(U_{n}\right) d U_{n},
$$

где $E_{n}$ - полная энергия электрона, $U_{n}-$ потенциальная энергия электрона, $E_{n}-U_{n}=E_{\mathrm{kin}}-$ кинетическая энергия электрона $c$-зоны, $\mathscr{P}\left(U_{n}\right)$ - плотность распределения вероятностей флуктуаций потенциальной энергии электрона. Тогда средняя по объему $V$ кристаллического образца концентрация электронов в $c$-зоне (при полной ионизации атомов водородоподобных примесей) равна

$$
n^{(\mathrm{re})}=\frac{1}{V} \int_{-\infty}^{+\infty} g_{n}^{(\mathrm{re})}\left(E_{n}\right) f_{n}\left(E_{n}\right) d E_{n}=(1-K) N,
$$

где $f_{n}\left(E_{n}\right)=\left\{1+\exp \left[\left(E_{n}-E_{\mathrm{F}}^{(c)}\right) / k_{\mathrm{B}} T\right]\right\}^{-1}-$ функция Ферми-Дирака, $E_{\mathrm{F}}^{(c)}-$ уровень Ферми.

Далее, следуя [24,25], считаем

$$
\mathscr{P}\left(U_{n}\right)=\frac{1}{\sqrt{2 \pi} W_{n}} \exp \left(-\frac{U_{n}^{2}}{2 W_{n}^{2}}\right),
$$

где $W_{n}$ - среднеквадратичная флуктуация энергии электрона $c$-зоны (рис. 1). При $n^{(\text {re) }}=(1-K) N$ величина $W_{n}$ связана со среднеквадратичной флуктуацией электростатической энергии неподвижного иона примеси $W_{d}$ соотношением $[25,26]$

$$
W_{n} \approx 0.21(1-K)^{1 / 2} W_{d} .
$$

При учете кулоновского взаимодействия только ближайших по расстоянию точечных заряженных частиц (ионов примесей и электронов) для $W_{d}$ имеем $[17,25]$

$$
W_{d} \approx 2.64 \frac{e^{2}}{4 \pi \varepsilon} N_{\mathrm{ch}}^{1 / 3},
$$

где $N_{\mathrm{ch}}=N_{+1}+K N+n^{(\mathrm{re})}=2 N-$ концентрация всех точечных частиц (с зарядом $\pm e$ ), удовлетворяющая условию электрической нейтральности полупроводника (6).

Отметим, что при $W_{n} \rightarrow 0$ плотность распределения вероятностей электростатических флуктуаций энергии электронов $c$-зоны $\mathscr{P}\left(U_{n}\right) \rightarrow \delta\left(U_{n}\right)$, где $\delta\left(U_{n}\right)-$ $\delta$-функция Дирака, и формула (6) переходит в (3).

Из формулы (6) определим концентрацию подвижных электронов $c$-зоны $n_{\text {mob }}$ с энергией $E_{n}=E_{\mathrm{kin}}+U_{n}$, большей энергии $E_{\mathrm{mob}}^{(c)}$, необходимой для их миграции в пределах всего трехмерного кристаллического re-полупроводника:

$$
\begin{aligned}
n_{\mathrm{mob}} & =\frac{\left(2 m_{n d}\right)^{3 / 2}}{2 \pi^{2} \hbar^{3}} \int_{E_{\mathrm{mob}}^{(c)}}^{\infty} f_{n} \int_{-\infty}^{E_{n}} \sqrt{E_{n}-U_{n}} \mathscr{P}\left(U_{n}\right) d U_{n} d E_{n} \\
& =n^{(\mathrm{re})}-n_{\mathrm{loc}},
\end{aligned}
$$

где $n_{\text {loc }}-$ концентрация электронов, которые могут двигаться только в ограниченных участках образца.

Входящая в выражение (10) величина $E_{\mathrm{mob}}^{(c)}<0-$ порог миграции (или „протекания“) электронов $c$-зоны $[17,26] ; E_{\text {mob }}^{(c)}$ обозначается так же, как $E_{\text {per, }}^{(c)}$ т.е. как энергия протекания (перколяции) электронной жидкости. Следуя $[23,27,28]$, полагаем, что критическая доля трехмерного полупроводникового образца, недоступная для миграции сквозь весь образец электронов $c$-зоны и соответствующая уровню энергии их протекания $\left(E_{n} \leqslant E_{\mathrm{mob}}^{(c)}\right)$, равна 0.17 . Тогда при учете (7) имеем

$$
\begin{aligned}
\int_{-\infty}^{E_{\text {mob }}^{(c)}} \mathscr{P}\left(U_{n}\right) d U_{n} & =\frac{1}{\sqrt{2 \pi} W_{n}} \int_{-\infty}^{E_{\text {mob }}^{(c)}} \exp \left(-\frac{U_{n}^{2}}{2 W_{n}^{2}}\right) d U_{n} \\
& =0.17,
\end{aligned}
$$

откуда получаем

$$
E_{\mathrm{mob}}^{(c)} \approx-0.955 W_{n}<0,
$$

где $W_{n}$ дается формулой (8) при учете (9).

\section{3. Расчет электрической проводимости и дрейфовой подвижности при упругом рассеянии электронов на ионах примесей в реальных полупроводниках}

Согласно $[16,22,29,30]$, статическая (на постоянном токе) электропроводность идеально (id) легированного 
полупроводника $n$-типа при учете формулы (3) имеет вид

$$
\begin{aligned}
\sigma_{n}^{(\mathrm{id})}= & \frac{2 e^{2}}{3 m_{n \sigma} V k_{\mathrm{B}} T} \\
& \times \int_{E_{c}=0}^{\infty} \tau_{n}^{(\mathrm{id})}\left(\varepsilon_{\mathrm{kin}}\right) g_{n}^{(\mathrm{id})}\left(\varepsilon_{\text {kin }}\right) f_{n}\left(1-f_{n}\right) \varepsilon_{\text {kin }} d \varepsilon_{\text {kin }} \\
\equiv & e n^{(\mathrm{id})} \mu_{n}^{(\mathrm{id})},
\end{aligned}
$$

где $m_{n \sigma}$ - эффективная масса электропроводности электрона, $\tau_{n}^{(\text {id) }}\left(\varepsilon_{\text {kin }}\right)$ - время релаксации квазиимпульса электрона (с кинетической энергией $\left.\varepsilon_{\mathrm{kin}}\right), \mu_{n}^{(\mathrm{id})}$ - дрейфовая подвижность;

$$
f_{n}\left(1-f_{n}\right)=\left\{4 \mathrm{ch}^{2}\left[\left(\varepsilon_{\text {kin }}-\varepsilon_{\mathrm{F}}^{(c)}\right) / 2 k_{\mathrm{B}} T\right]\right\}^{-1} .
$$

Исходя из формулы (12) определим электропроводность ге-полупроводника $\sigma_{n}^{(\text {re) }}=\sigma_{n}$. Для этого в (12) заменим плотность электронных состояний $g_{n}^{(\mathrm{id})}\left(\varepsilon_{\mathrm{kin}}\right)$ из (3) на $g_{n}^{(\text {re) }}\left(E_{n}\right)$ по (5), дно $c$-зоны $E_{c}=0-$ на $E_{\text {mob }}^{(c)}<0$, а также время релаксации $\tau_{n}^{(\text {id })}\left(\varepsilon_{\text {kin }}\right)-$ на $\tau_{\text {ion }}^{(\text {re })}\left(E_{n}-U_{n}\right)=\tau_{\text {ion }}^{(\text {re })}\left(E_{\text {kin }}\right)$. В итоге статическая электропроводность реально (re) легированного полупроводника $n$-типа при учете (5)-(11) может быть представлена в виде

$$
\begin{aligned}
\sigma_{n}= & \sigma_{n}^{(\mathrm{re})}=\frac{e^{2}}{m_{n \sigma}} \frac{\left(2 m_{n d}\right)^{3 / 2}}{3 \pi^{2} \hbar^{3}} \int_{E_{\mathrm{mob}}^{(c)}}^{\infty} \frac{f_{n}\left(1-f_{n}\right)}{k_{\mathrm{B}} T} \\
& \times \int_{-\infty}^{E_{n}} \tau_{\mathrm{ion}}^{(\mathrm{re})}\left(E_{n}-U_{n}\right)\left(E_{n}-U_{n}\right)^{3 / 2} \mathscr{P}\left(U_{n}\right) d U_{n} d E_{n},
\end{aligned}
$$

где $f_{n}\left(1-f_{n}\right)=\left\{4 \mathrm{ch}^{2}\left[\left(E_{n}-E_{\mathrm{F}}^{(c)}\right) / 2 k_{\mathrm{B}} T\right]\right\}^{-1}$.

Входящее в (13) время релаксации квазиимпульса электрона с кинетической энергией $E_{\text {kin }}=E_{n}-U_{n}>0$ (при $\left.E_{n}>E_{\mathrm{mob}}^{(c)}\right)$ при упругом рассеянии на ионах водородоподобных примесей в кристаллической матрице с диэлектрической проницаемостью $\varepsilon=\varepsilon_{r} \varepsilon_{0}$, согласно $[18,19]$, есть

$$
\begin{aligned}
& \tau_{\text {ion }}^{(\mathrm{re})}\left(E_{n}-\right.\left.U_{n}\right)=\left(\frac{4 \pi}{3}\right)^{1 / 3}\left(\frac{4 \pi \varepsilon}{e^{2}}\right)^{2} \frac{\hbar\left(E_{n}-U_{n}\right)}{N_{\text {ion }}^{2 / 3}} \\
& \times \ln ^{-1}\left[1+\left(\frac{8 \pi \varepsilon R_{\text {ion }}\left(E_{n}-U_{n}\right)}{e^{2}}\right)^{2}\right],
\end{aligned}
$$

где $N_{\text {ion }}=(1+K) N$, величина $R_{\text {ion }}$ определяется из формулы (2). Отметим, что при получении формулы (14) учитывалось только кулоновское взаимодействие электрона с ионом, расположенным в центре шара объемом $1 / N_{\text {ion. }}$ Считалось, что локальная статическая относительная диэлектрическая проницаемость матрицы $\varepsilon_{r}$ в окрестности каждого иона примеси определяется электронами $v$-зоны.

Дрейфовая подвижность электронов $c$-зоны, ограниченная их упругим рассеянием на ионах примесей, в re-полупроводнике $n$-типа определяется по аналогии с формулой (12) как

$$
\mu_{n}=\mu_{\mathrm{ion}}^{(\mathrm{re})}=\frac{\sigma_{n}^{(\mathrm{re})}}{e n_{\mathrm{mob}}}=\frac{\sigma_{n}}{e n_{\mathrm{mob}}},
$$

где $\sigma_{n}=\sigma_{n}^{(\mathrm{re})}$ определяется по (13) с учетом (14), a $n_{\text {mob }}$ по (10).

Сопоставим формулы (13) и (15) для re-полупроводника с вырожденным газом электронов в $c$-зоне. С одной стороны, согласно формуле (13), в электрической проводимости re-полупроводника на постоянном токе участвуют электроны, энергия $E_{n}$ которых превышает порог подвижности $E_{\mathrm{mob}}^{(c)}$. При этом в актах рассеяния на ионах примесей эффективно участвуют только те электроны $c$-зоны, полная энергия которых $E_{n}$ близка к уровню Ферми $E_{\mathrm{F}}^{(c)}$. (Формально это обусловлено тем обстоятельством, что функция $f_{n}\left(1-f_{n}\right)$ под знаком интеграла в (13) имеет острый максимум при $E_{n}=E_{\mathrm{F}}^{(c)}$.) С другой стороны, при записи формулы (15) полагается, что величину $\sigma_{n}=e n_{\text {mob }} \mu_{n}$ определяют все подвижные электроны с концентрацией $n_{\mathrm{mob}}$, но, как будто, все они имеют одинаковую подвижность $\mu_{n}$ (и дрейфовую скорость). Итак, при записи формул (13) и (15) принималось, что статическая электропроводность re-полупроводника с вырожденным газов электронов в $c$-зоне равна произведению элементарного заряда, концентрации всех подвижных электронов и дрейфовой подвижности электронов с полной энергией в окрестности уровня Ферми. Это согласуется с общепринятой теорией электронной проводимости полупроводников $[16,22,30,31]$, но не согласуется с представлениями, развиваемыми в [32].

Отметим, что в пренебрежении электростатическими флуктуациями потенциальной энергии электронов $\left(W_{n} \ll k_{\mathrm{B}} T\right)$, когда $\mathscr{P}\left(U_{n}\right) \rightarrow \delta\left(U_{n}\right)$ и $\left|E_{\text {mob }}^{(c)}\right| \ll k_{\mathrm{B}} T$, согласно (10) имеем $n_{\text {loc }} \rightarrow 0$ и $n_{\text {mob }} \approx n^{(\mathrm{re})} \approx n^{(\mathrm{id})}$. При этом формулы (13) и (15) переходят в стандартные формулы $(12)$ и $\mu_{n}^{\text {(id) }}=\mu_{\text {ion }}^{(\text {(id) }}=\sigma_{n}^{\text {(id) }} / e n^{(\text {id) }}$ для id-полупроводника [16,22,30,31].

Опишем алгоритм расчетов по формулам (6)-(15) на примере полупроводника $n$-типа (см. также $[25,26])$ :

1) задаются исходные параметры полупроводника $n$-типа: $\varepsilon_{r}, m_{n d}, m_{n \sigma}$, температура $T$, диапазон изменения концентрации водородоподобных доноров $N$, степень компенсации доноров водородоподобными акцепторами $K$ и относительная погрешность вычислений $\varepsilon_{\text {goal }}=10^{-5}$;

2) по формулам (8) и (9), где $N_{\text {ch }}=2 N$, рассчитываются значения среднеквадратичных флуктуаций электростатической потенциальной энергии электронов и ионов $W_{n}$ и $W_{d}$;

$3)$ численными методами решается нелинейное уравнение электрической нейтральности (6) относительно неизвестного уровня Ферми $E_{\mathrm{F}}^{(c)}$ с учетом полученных значений $W_{n}$ и $W_{d}$; 
4) по формуле (11) на основе полученного $W_{n}$ вычисляется $E_{\mathrm{mob}}^{(c)}$. Затем по формуле (10) вычисляется $n_{\mathrm{mob}}$;

5) по (13) с учетом (14) и (2) рассчитывается электропроводность $\sigma_{n}=\sigma_{n}^{(\mathrm{re})}$;

6) наконец, по формуле (15) рассчитывается подвижность $\mu_{n}=\mu_{\text {ion }}^{(\mathrm{re})}$.

\section{4. Модель Конуэлл-Вайскопфа (CW) для дрейфовой подвижности электронов в идеальных полупроводниках}

Для сравнения с формулами (13) и (15) приведем здесь необходимые формулы модели $\mathrm{CW}$ для идеального вырожденного газа электронов $c$-зоны id-полупроводника (см. также формулы (3) и (12)). Согласно этой модели, время релаксации квазиимпульса электрона $c$-зоны с кинетической энергией $\varepsilon_{\text {kin }}$ при упругом рассеянии на водородоподобных ионах примесей (см., например, [33-35]) есть

$$
\begin{aligned}
\tau_{n}^{(\mathrm{id})}\left(\varepsilon_{\text {kin }}\right)= & \tau_{\mathrm{CW}}\left(\varepsilon_{\text {kin }}\right)=\left(\frac{4 \pi \varepsilon}{e^{2}}\right)^{2} \frac{\left(2 m_{\text {nd }}^{(1)}\right)^{1 / 2} \varepsilon_{\text {kin }}^{3 / 2}}{\pi N_{\text {ion }}} \\
& \times \ln ^{-1}\left[1+\left(\frac{4 \pi \varepsilon \varepsilon_{\text {kin }}}{e^{2} N_{\text {ion }}^{1 / 3}}\right)^{2}\right],
\end{aligned}
$$

где $\varepsilon_{\text {kin }}-$ кинетическая энергия электрона $\left(\varepsilon_{\text {kin }}=E_{n}\right.$ при $W_{n}=E_{\text {mob }}^{(c)}=0$; см. также рис. 1$), N_{\text {ion }}=(1+K) N-$ концентрация ионов примесей. (Отметим, что при получении формулы (16) время взаимодействия электрона с одним ионом примеси формально принималось бесконечно большим.)

Согласно $[30,34,36]$, дрейфовая подвижность электронов в модели CW определяется из формулы (12) как

$$
\mu_{\mathrm{CW}}=\mu_{\mathrm{ion}}^{(\mathrm{id})}=\frac{\sigma_{n}^{(\mathrm{id})}}{e n^{(\mathrm{id})}}=\frac{e\left\langle\tau_{\mathrm{CW}}\right\rangle}{m_{n \sigma}},
$$

где среднее время релаксации квазиимпульса электрона $c$-зоны (при $W_{n}=E_{\mathrm{mob}}^{(c)}=0$; рис. 1) равно

$$
\begin{aligned}
\left\langle\tau_{\mathrm{CW}}\right\rangle= & \int_{E_{c}=0}^{\infty} \tau_{\mathrm{CW}}\left(\varepsilon_{\mathrm{kin}}\right) \varepsilon_{\mathrm{kin}}^{3 / 2} f_{n}\left(1-f_{n}\right) d \varepsilon_{\mathrm{kin}} \\
& \times\left[\int_{E_{c}=0}^{\infty} \varepsilon_{\mathrm{kin}}^{3 / 2} f_{n}\left(1-f_{n}\right) d \varepsilon_{\mathrm{kin}}\right]^{-1} .
\end{aligned}
$$

Электропроводность $\sigma_{\mathrm{CW}}$ дается формулой при $\tau_{n}^{(\mathrm{id})}\left(\varepsilon_{\mathrm{kin}}\right)=\tau_{\mathrm{CW}}\left(\varepsilon_{\mathrm{kin}}\right)$ и связана с дрейфовой подвижностью электронов $\mu_{\mathrm{CW}}$ стандартным выражением $[33,34,36]$ :

$$
\sigma_{\mathrm{CW}}=\sigma_{n}^{(\mathrm{id})}=e n^{(\mathrm{id})} \mu_{\mathrm{CW}},
$$

где $n^{(\mathrm{id})}=(1-K) N-$ концентрация электронов в $c$-зоне id-полупроводника по формуле (3), определяет значение уровня Ферми $\varepsilon_{\mathrm{F}}^{(c)}$ в формуле (18).

Отметим, что все записанные в разд. 3 и 4 формулы справедливы и для полупроводника $p$-типа, содержащего ионы водородоподобных акцепторов и доноров, при замене в них эффективной массы плотности состояний $m_{n d}$ на $m_{p d}$, эффективной массы электропроводности $m_{n \sigma}$ на $m_{p \sigma}$ и средней концентрации электронов $c$-зоны $n$ на среднюю концентрацию дырок $v$-зоны $p$.

\section{5. Сравнение результатов расчетов с экспериментальными данными}

Для сравнения расчетов электропроводности на постоянном токе $\sigma_{n}$ и дрейфовой подвижности $\mu_{n}$ с данными экспериментов выбирались сильно легированные кристаллы германия, кремния, арсенида галлия и антимонида индия $n$-типа. У этих полупроводников концентрация электронов $n=n^{(\mathrm{id})}=(1-K) N$ по (3) удовлетворяла неравенству (4), а концентрация доноров $N$ была больше моттовской $N_{\mathrm{M}}$, определяемой из соотношения (1). Отметим, что численный расчет по (10) дает для вырожденных кристаллов $n$-типа отношение $n_{\mathrm{loc}} / n^{(\mathrm{re})}<0.2$ (для $\mathrm{Si}, \mathrm{Ge}$ ),$n_{\mathrm{loc}} / n^{(\mathrm{re})}<0.02$ (для $\mathrm{GaAs}, \mathrm{InSb})$ при $n^{(\mathrm{re})}>n_{\mathrm{M}}$, где моттовская концентрация электронов $n_{\mathrm{M}}=(1-K) N_{\mathrm{M}}$. Это обстоятельство позволяет заключить, что концентрация $n_{\mathrm{mob}}=n^{(\mathrm{re})}-n_{\mathrm{loc}}$ свободных (в пределах всего кристаллического образца) электронов $c$-зоны практически равна их средней концентрации в образце. Этот вывод согласуется с аналитическими оценками [37,38], отождествляющими измеряемую на постоянном токе в сильном магнитном поле холловскую концентрацию основных носителей заряда с их средней концентрацией в трехмерных частично разупорядоченных кристаллических полупроводниках. Электроны $c$-зоны с концентрацией $n_{\mathrm{loc}}$ напрямую не участвуют в электрической проводимости и эффекте Холла на постоянном токе, но вносят вклад в макроскопическую статическую диэлектрическую проницаемость полупроводника.

На рис. 2-5 представлены экспериментальные и расчетные зависимости электропроводности на постоянном токе, холловской (эксперимент) и дрейфовой (расчет по предложенному алгоритму) подвижностей электронов $c$-зоны, а также уровня Ферми и порога миграции $E_{\mathrm{mob}}^{(c)}$ от концентрации легирующих примесей $N$ и электронов $n=(1-K) N$. Рассмотрены слабо компенсированные $(K \ll 1)$ кристаллы $n$-Ge (рис. 2) и $n$-Si (рис. 3), а также умеренно компенсированные $(K \approx 0.1)$ кристаллы $n$-GaAs и $n$-InSb при криогенных температурах (рис. 4 и 5). Сплошные линии на рис. 2-5 вычислены по формулам (6), (10), (13) и (15) согласно развитой нами квазиклассической модели равновесных состояний и рассеяния электронов, штриховые кривые - расчет $\mu_{\mathrm{CW}}$ и $\sigma_{\mathrm{CW}}$ по формулам (19) и (17) согласно модели CW $[33,39]$. 


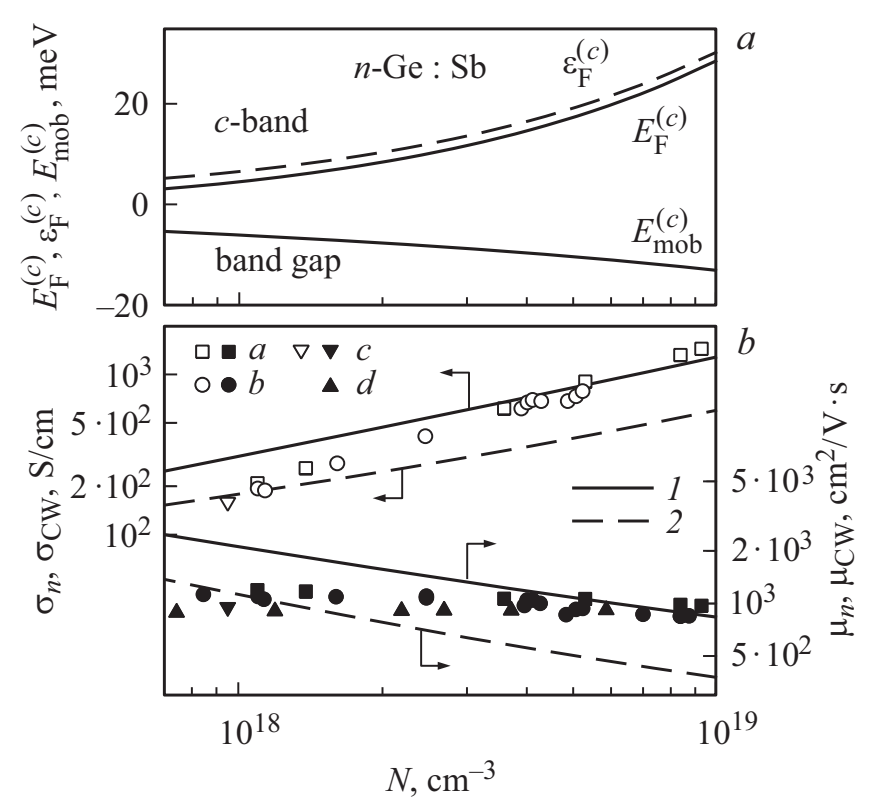

Рис. 2. $a-$ расчетные зависимости положения уровня Ферми $E_{\mathrm{F}}^{(c)}$ и порога миграции электронов $E_{\mathrm{mob}}^{(c)}$ по формулам (6) и (11) от концентрации атомов сурьмы (как водородоподобных доноров) $N$ в кристаллах $n$-Ge:Sb при степени компенсации $K=0.01$, температуре $T=3 \mathrm{~K}$ и концентрации электронов $c$-зоны $n=(1-K) N$. Штриховая линия - расчет $\varepsilon_{\mathrm{F}}^{(c)}$ по формуле (3) для идеального ферми-газа. $b-$ зависимости статической электропроводности $\sigma_{n}$ и холловской подвижности $\mu_{n}$ электронов от концентрации атомов сурьмы. Данные измерений, $T, \mathrm{~K}: a-4.2[40], b-1.3[9,10], c-2.5[41]$, $d-4.2$ [42]. Линии - расчет для $K=0.01, T=3 \mathrm{~K}$ и $n=(1-K) N: 1-\sigma_{n}$ по (13), дрейфовая подвижность $\mu_{n}$ по $(15) ; 2-\sigma_{\mathrm{CW}}$ по (19) и $\mu_{\mathrm{CW}}$ по (17).

Точками показаны экспериментальные данные для $n-\mathrm{Ge}: \mathrm{Sb}$ из работ $[9,10,40-42]$ (см. также $[39,43,44]$ ), для $n$-Si:P $[11,12,45] \quad$ (см. также $[39,43,46])$, для $n$-GaAs:Sn [47,48], n-GaAs:Te [49,50] и n-GaAs [51], а также для $n$-InSb:Se [52], $n$-InSb:Sn [53,54] и $n$-InSb:Te [55].

Здесь отметим, что холловская подвижность электронов в предельно чистых кристаллах $\mathrm{Ge}, \mathrm{Si}, \mathrm{GaAs}$ и InSb при температуре $T \approx 78 \mathrm{~K}$, когда доминирует рассеяние на фононах (подвижность $\left.\mu_{\mathrm{lat}}\right)$, согласно [56,57], составляет: $\mu_{\text {lat }} \approx 4 \cdot 10^{4} \mathrm{~cm}^{2} / \mathrm{B} \cdot$ с для $n$-Ge, $\mu_{\text {lat }} \approx 2 \cdot 10^{4} \mathrm{~cm}^{2} / \mathrm{B} \cdot$ с для $n-\mathrm{Si}, \mu_{\text {lat }} \approx 2 \cdot 10^{5} \mathrm{~cm}^{2} / \mathrm{B} \cdot \mathrm{c}$ для $n$-GaAs и $\mu_{\text {lat }} \approx 10^{6} \mathrm{~cm}^{2} / \mathrm{B} \cdot$ с для $n$-InSb. Вследствие того что $\mu_{\text {lat }} \gg \mu_{n}$ (см. рис. $\left.2, b, 3, b, 4, b, 5, b\right)$, рассеяние на фононах при криогенных температурах не учитывалось.

При расчетах уровней электропроводности и дрейфовой подвижности электронов в германии и кремнии использовались следующие значения физических величин [56,57]: для $n$-Ge (число долин в $c$-зоне $v=4): \varepsilon_{r}=15.4, m_{n d}=0.554 m_{0}, m_{n \sigma}=0.119 m_{0}$; для $n$-Si $(v=6): \varepsilon_{r}=11.47, m_{n d}=1.062 m_{0}, m_{n \sigma}=0.259 m_{0}$, где $m_{0}$ - масса электрона в вакууме. (Зависимостью эффективной массы электрона от концентрации элек-

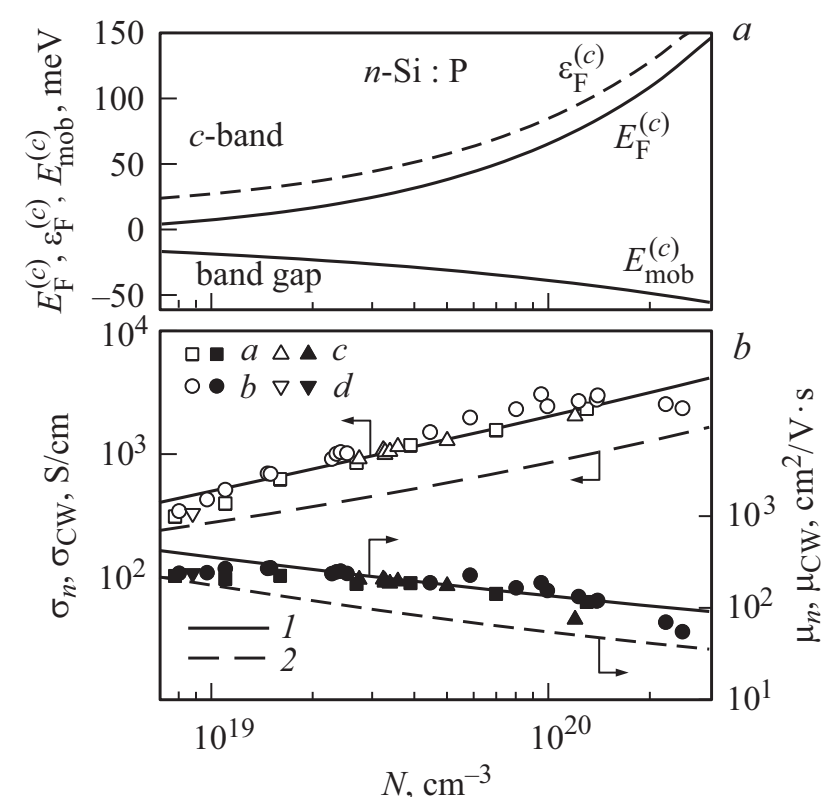

Рис. 3. $a-$ расчетные зависимости уровня Ферми $E_{\mathrm{F}}^{(c)}$ и порога миграции электронов $E_{\mathrm{mob}}^{(c)}$ по формулам (6) и (11) от концентрации атомов фосфора (как водородоподобных доноров) $N$ в кристаллах $n$-Si:P при степени компенсации $K=0.01$, температуре $T=3 \mathrm{~K}$ и концентрации электронов $c$-зоны $n=(1-K) N$. Штриховая линия - расчет $\varepsilon_{\mathrm{F}}^{(c)}$ по формуле (3) для идеального ферми-газа. $b-$ зависимости статической электропроводности $\sigma_{n}$ и холловской подвижности $\mu_{n}$ электронов от концентрации атомов фосфора. Данные измерений, $T, \mathrm{~K}: a-4.2[11], b-4.2[12], c-78$ [12], $d-4.2$ [45]. Линии - расчет при $K=0.01, T=3 \mathrm{~K}$ и $n=(1-K) N: 1-\sigma_{n}$ по $(13)$ и дрейфовой подвижности $\mu_{n}$ по (15), $2-\sigma_{\mathrm{CW}}$ по (19) и $\mu_{\mathrm{CW}}$ по (17).

тронов для $n-\mathrm{Ge}\left(\right.$ до $3 \cdot 10^{19} \mathrm{~cm}^{-3}$ ) и $n-\mathrm{Si}\left(\right.$ до $3 \cdot 10^{20} \mathrm{~cm}^{-3}$ ) можно пренебречь [56,57].)

Для сильно легированных вырожденных кристаллов $n$-GaAs $\quad\left(v=1 ; \quad \varepsilon_{r}=12.4\right)$ и $n$-InSb $\quad\left(v=1 ; \quad \varepsilon_{r}=16.8\right)$ необходимо учитывать зависимость эффективных масс $m_{n d}=m_{n \sigma} \quad$ электронов $\quad$ от $\quad$ их $\quad$ концентрации c-зоне [56,57]:

$$
n \text {-GaAs : } \quad m_{n d}(n)=m_{n d}\left[1+6.4 \cdot 10^{-4}\left(n / n_{*}\right)^{0.645}\right] \text {, }
$$

где $m_{n d}=m_{n \sigma}=0.067 m_{0}, n_{*}=1 \cdot 10^{15} \mathrm{~cm}^{-3}$;

$$
n \text {-InSb : } \quad m_{n d}(n)=m_{n d}\left[1+2.6 \cdot 10^{-2}\left(n / n_{*}\right)^{0.3}\right],
$$

где $m_{n d}=m_{n \sigma}=0.013 m_{0}, n_{*}=3.1 \cdot 10^{12} \mathrm{~cm}^{-3}$.

Из рис. $2, b, 3, b, 4, b$ и $5, b$ видно, что в целом наблюдается согласие расчетов по формулам (13) и (15) с экспериментальными данными для кристаллов $n-\mathrm{Ge}: \mathrm{Sb}$, $n$-Si:P, $n$-GaAs и $n$-InSb.

Для слабо компенсированных кристаллов $n-\mathrm{Ge}: \mathrm{Sb}$ при концентрации сурьмы $N<10^{18} \mathrm{~cm}^{-3}$ наблюдается некоторое расхождение результатов расчета по (13) и (15) с экспериментом. Это, возможно, связано с 
наличием упругих деформаций кристаллической решетки в окрестности атомов $\mathrm{Sb}$, которые сглаживаются при высоких значениях концентрации этой легирующей примеси [58-60].

Относительно подвижности электронов в $n$-GaAs (рис. 4) отметим, что уменьшение $\mu_{n}$ при $n \approx 10^{19} \mathrm{~cm}^{-3}$, возможно, обусловлено небольшим увеличением степени компенсации при увеличении концентрации легирующей примеси как для $n$-GaAs: $\mathrm{Sb}$, так и для $n$-GaAs:Te (см., например, $[13,61]$ ).

Относительно подвижности электронов в $n$ - InSb (рис. 5) отметим, что в этом полупроводнике с малой эффективной массой электрона возможно его рассеяние не на одном (как в предлагаемой нами модели расчета времени релаксации), а на двух ионах примесей одновременно [62]. Вследствие этого подвижность электронов будет несколько выше, чем при рассеянии на одном центре (ионе).

Из рис. 2, $a, 3, a, 4, a$ и 5, $a$ видно, что увеличение концентрации основных примесей (и электронов)

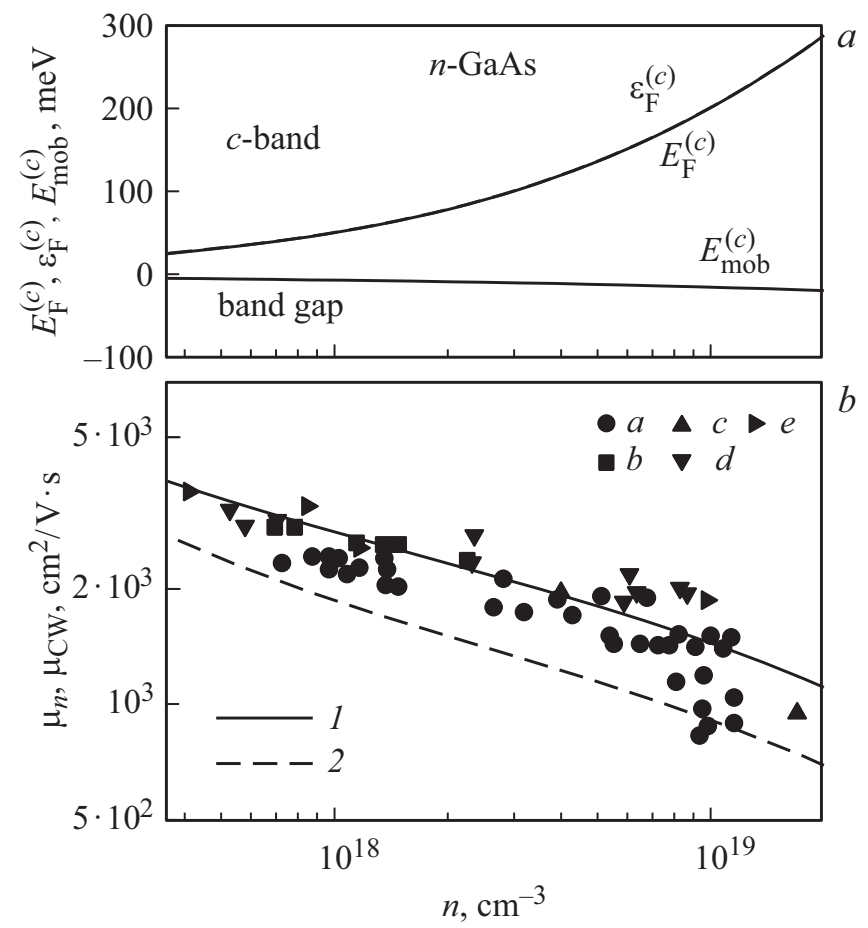

Рис. 4. $a$ - расчетные зависимости уровня Ферми $E_{\mathrm{F}}^{(c)}$ и порога миграции электронов $E_{\text {mob }}^{(c)}$ в кристаллах $n$-GaAs по формулам (6) и (11) от концентрации электронов $n=(1-K) N$ для степени компенсации $K=0.1$, температуры $T=78 \mathrm{~K}$ и концентрации водородоподобных доноров $N$. Штриховая линия расчет $\varepsilon_{\mathrm{F}}^{(c)}$ по формуле (3) для идеального ферми-газа при учете зависимости $m_{n d}=m_{n \sigma}$ от $n$ по (20). $b$ - зависимость холловской подвижности электронов $\mu_{n}$ от их концентрации в $n$-GaAs при температуре $T=78 \mathrm{~K}$. Данные измерений: $a-n$-GaAs:Sn [47], $b-n$-GaAs:Sn [48], $c-n$-GaAs:Te [49]; $d-n$-GaAs:Te [50]; $e-n$-GaAs [51]. Линии - расчет для $K=0.1, T=78 \mathrm{~K}$ и $n=(1-K) N$ при учете $(20): 1-$ дрейфовой подвижности $\mu_{n}$ по (15), $2-\mu_{\mathrm{CW}}$ по (17).

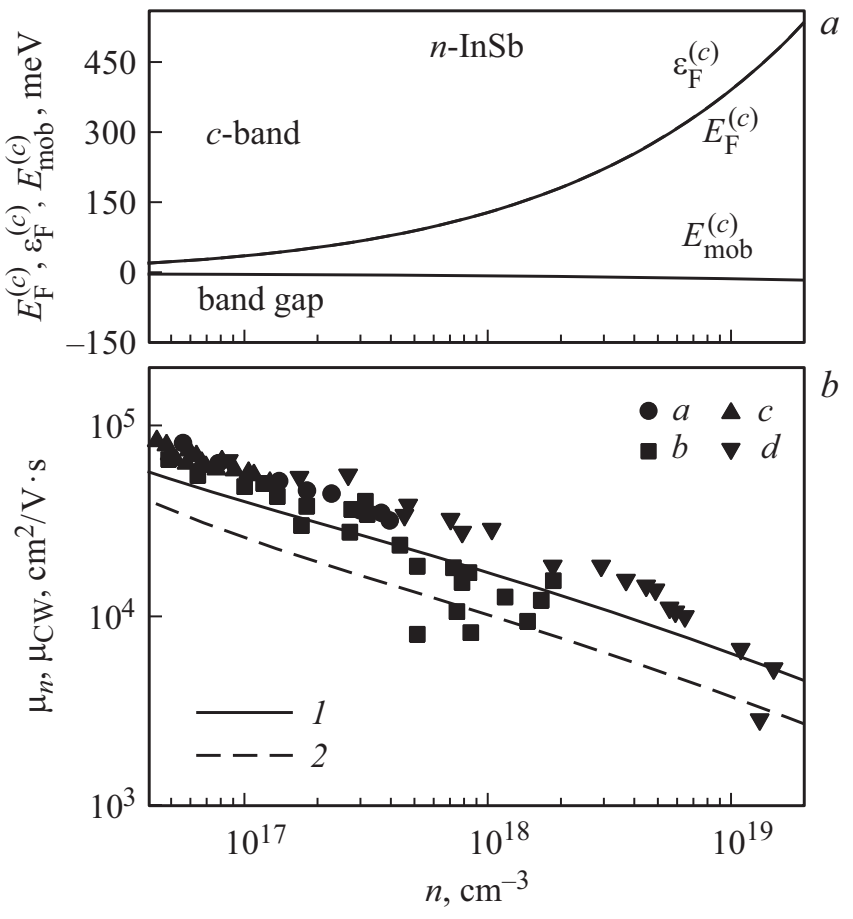

Рис. 5. $a-$ расчетные зависимости уровня Ферми $E_{\mathrm{F}}^{(c)}$ и порога миграции электронов $E_{\mathrm{mob}}^{(c)}$ в кристаллах $n$-InSb по формулам (6) и (11) от концентрации электронов $n=(1-K) N$ для степени компенсации $K=0.1$, температуры $T=78 \mathrm{~K}$ и концентрации водородоподобных доноров $N$. Штриховая линия - расчет $\varepsilon_{\mathrm{F}}^{(c)}$ по формуле (3) для идеального фермигаза при учете зависимости $m_{n d}=m_{n \sigma}$ от $n$ по (21). $b-$ зависимость холловской подвижности $\mu_{n}$ электронов от их концентрации в $n$-InSb при температуре $T=78 \mathrm{~K}$. Данные измерений: $a-n-\mathrm{InSb}: \mathrm{Se}$ [52], $b-n-\mathrm{InSb}: \mathrm{Sn}$ [53], $c-n-\mathrm{InSb}: \mathrm{Sn}$ [54], $d-n$-InSb:Te [55]. Линии - расчет при $K=0.1, T=78 \mathrm{~K}$ и $n=(1-K) N$ при учете $(21): 1-$ дрейфовой подвижности $\mu_{n}$ по (15), $2-\mu_{\mathrm{CW}}$ по (17).

сдвигает уровень Ферми $E_{\mathrm{F}}^{(c)}$ (сплошная линия) для re-полупроводников глубже в $c$-зону по сравнению с уровнем Ферми $\varepsilon_{\mathrm{F}}^{(c)}$ (штриховая линия) для id-полупроводников. При прочих равных условиях чем меньше значение эффективной массы электрона $m_{n d}$, тем меньше различие между $\varepsilon_{\mathrm{F}}^{(c)}$ и $E_{\mathrm{F}}^{(c)}$.

\section{6. Обсуждение}

При получении и формулы (14) в [18], и формулы (16) в [33] взаимодействие электрона при его рассеянии ионом примеси принималось чисто кулоновским. В модели электрон-ионного рассеяния по БруксуХеррингу $[16,22,31,39,63]$ кулоновский потенциал взаимодействия электрона $c$-зоны с ионом примеси заменяется экранированным кулоновским потенциалом (в приближении Дебая-Хюккеля или Томаса-Ферми для невырожденного и вырожденного газа электронов соответственно). Однако, согласно [64,65], экранированный 
кулоновский потенциал (т. е. суммарный потенциал иона и облака экранирующих его зарядов) нельзя трактовать как потенциал парного взаимодействия электрона и иона.

Заметим, что согласно данным, представленным на рис. $2, b, 3, b, 4, b$ и $5, b$, ограничение дрейфовой подвижности рассеянием электронов $c$-зоны на ионах примеси при криогенных температурах является доминирующим по сравнению с рассеянием на фононах. В связи с этим вкратце рассмотрим еще электрон-электронное и резонансное рассеяния электронов на ионах доноров в рамках модели id-полупроводника (см. формулы (3), (4) и (12)).

Для оценки подвижности, ограниченной рассеянием электронов на электронах в $c$-зоне вырожденного id-полупроводника, исходим из формулы для среднего времени релаксации $\tau_{e e}$ по [66] (см. также [67]):

$$
\frac{1}{\tau_{e e}}=\frac{\pi}{8} \frac{\left(k_{\mathrm{B}} T\right)^{2}}{\hbar \varepsilon_{\mathrm{F}}^{(c)}}+\frac{\sqrt{3}}{2 \hbar \sqrt{\varepsilon_{\mathrm{F}}^{(c)}}}\left(\frac{k_{\mathrm{B}} T}{k_{\mathrm{F}} l_{e e}}\right)^{3 / 2},
$$

где $l_{e e}=v_{\mathrm{F}} \tau_{\mathrm{CW}}\left(\varepsilon_{\mathrm{kin}}\right)$ - длина свободного пробега электрона с кинетической энергией $\varepsilon_{\mathrm{kin}} \approx \varepsilon_{\mathrm{F}}^{(c)} \pm k_{\mathrm{B}} T$ и скоростью Ферми $v_{\mathrm{F}} \approx\left[2 \varepsilon_{\mathrm{F}}^{(c)} / m_{n d}^{(1)}\right]^{1 / 2}$ при рассеянии на электроне; $k_{\mathrm{F}} \approx\left[3 \pi^{2} n^{(\mathrm{id})}\right]^{1 / 3}$ - величина волнового вектора электрона с кинетической энергией $\varepsilon_{\mathrm{F}}^{(c)} \approx \xi_{\mathrm{F}}^{(c)}$ по формуле (4). Тогда подвижность электронов, ограниченная электрон-электронным рассеянием, есть: $\mu_{e e}=e \tau_{e e} / m_{n \sigma}$, где $\tau_{e e}$ дается формулой (22).

Оценим минимальное значение среднего времени релаксации $\tau_{\text {res }}$ квазиимпульса электронов $c$-зоны при резонансном рассеянии на ионах доноров в вырожденном id-полупроводнике (см., например, $[68,69])$. Условно считая уровни энергии доноров равномерно распределенными в $c$-зоне в окрестности $\varepsilon_{\mathrm{F}}^{(c)}$, можно оценить $1 / \tau_{\text {res }}$ как

$$
1 / \tau_{\mathrm{res}} \approx \frac{k_{\mathrm{B}} T}{\varepsilon_{\mathrm{F}}^{(c)}} N_{\mathrm{res}} v_{\mathrm{F}} \sigma_{\mathrm{res}},
$$

где $k_{\mathrm{B}} T / \varepsilon_{\mathrm{F}}^{(c)}$ - доля резонансно рассеиваемых электронов с кинетической энергией $\varepsilon_{\text {kin }} \approx \varepsilon_{\mathrm{F}}^{(c)} \pm k_{\mathrm{B}} T$ и скоростью $v_{\mathrm{F}} ; N_{\text {res }} \approx\left(k_{\mathrm{B}} T / \varepsilon_{\mathrm{F}}^{(c)}\right) N$ - доля доноров в зарядовом состоянии $(+1)$, резонансно рассеивающих электроны; $\sigma_{\text {res }} \approx \pi R_{\text {ion }}^{2}-$ сечение резонансного рассеяния электрона донором (оценка по модели твердой сферы [70]); $R_{\text {ion }} \approx 0.62[(1+K) N]^{-1 / 3}$. Тогда дрейфовая подвижность электронов, ограниченная резонансным рассеянием, есть: $\mu_{\text {res }}=e \tau_{\text {res }} / m_{n \sigma}$, где $\tau_{\text {res }}$ дается формулой (23).

Наконец, общая дрейфовая подвижность электронов $\mu_{n}^{(\mathrm{id})}$ определяется по правилу Матиссена (см., например, [16,22,30]):

$$
1 / \mu_{n}^{(\mathrm{id})}=1 / \mu_{\mathrm{ion}}^{(\mathrm{id})}+1 / \mu_{e e}+1 / \mu_{\mathrm{res}} \approx 1 / \mu_{\mathrm{ion}}^{(\mathrm{id})},
$$

где вклад $\mu_{e e}$ и $\mu_{\text {res }}$ по (22) и (23) в общую подвижность $\mu_{n}^{\text {(id) }}$ составляет не более $3 \%$ от вклада $\mu_{\text {ion }}^{\text {(id) }}=\mu_{\mathrm{CW}}$ по (17).

\section{7. Заключение}

В работе развита квазиклассическая модель упругого рассеяния основных носителей заряда (электронов $c$-зоны) на неподвижных ионах водородоподобных примесей в сильно легированных вырожденных полупроводниках $n$-типа. Считалось, что в кристаллической матрице единичный акт упругого кулоновского рассеяния электрона на ионе происходит в течение времени пролета им сферической области (радиуса $\left.R_{\text {ion }} \approx 0.62[(1+K) N]^{-1 / 3}\right)$, в центре которой расположен один ион примеси при их общей концентрации $(1+K) N$, где $N-$ концентрация доноров, $K N-$ концентрация компенсирующих доноры акцепторов. Учтены электростатические флуктуации потенциальной энергии электронов $c$-зоны и, как следствие, смещение порога их миграции в глубь запрещенной энергетической зоны. На основании предложенного в данной работе алгоритма рассчитаны статическая (на постоянном токе) электропроводность и дрейфовая подвижность электронов при упругом рассеянии на ионах примесей при низких (криогенных) температурах. Показано, что вклад рассеяния подвижных электронов на фононах и электронах, а также резонансного рассеяния электронов на ионах доноров в общее рассеяние электронов в вырожденных полупроводниках $n$-типа при низких температурах не является определяющим.

Результаты расчетов по полученным в квазиклассическом приближении формулам без использования подгоночных параметров численно согласуются с известными экспериментальными данными для кристаллов германия, кремния, арсенида галлия и антимонида индия $n$-типа при достаточно высоких концентрациях доноров для низких и умеренных степеней их компенсации. В целом расчетные значения электропроводности и дрейфовой подвижности электронов по развитой нами модели больше, чем полученные в рамках модели КонуэллВайскопфа.

Работа выполнена при поддержке государственной программы „Маттех“ Республики Беларусь.

\section{Список литературы}

[1] Т.Т. Мнацаканов, М.Е. Левинштейн, Л.И. Поморцева, С.Н. Юрков. ФТП, 38 (1), 56 (2004).

[2] Г.Б. Лесовик, И.А. Садовский. УФН, 181 (10), 1041 (2011).

[3] В.Ф. Гантмахер. Физика низких температур, 39 (1), 5 (2013).

[4] Radiation Effects in Semiconductors, ed. by K. Iniewski (Boca Raton, CRC Press, 2011).

[5] A.H. Johnston. In: Proc. 4th Int. Workshop on Radiation Effects on Semiconductor Devices for Space Application (Tsukuba, Japan, October 11-13, 2000) p. 1.

[6] А.А. Лебедев, В.В. Козловский, Н.Б. Строкан, Д.В. Давыдов, А.М. Иванов, А.М. Стрельчук, Р. Якимова. ФТП, 36 (11), 1354 (2002). 
[7] B.J. Baliga. Fundamentals of Power Semiconductor Devices (Berlin, Springer, 2008).

[8] И.В. Грехов, Г.А. Месяц. УФН, 175 (7), 735 (2005).

[9] M. Cuevas, H. Fritzsche. Phys. Rev., 139 (5A), 1628 (1965).

[10] M. Cuevas, H. Fritzsche. Phys. Rev., 137 (6A), 1847 (1965).

[11] P.W. Chapman, O.N. Tufte, J.D. Zook, D. Long. J. Appl. Phys., 34 (11), 3291 (1963).

[12] C. Yamanouchi, K. Mizuguchi, W. Sasaki. J. Phys. Soc. Jpn., 22 (3), 859 (1967).

[13] В.М. Глазов. Матер. электрон. техники, № 2, 15 (1998).

[14] N.F. Mott. Metal-Insulator Transitions (London, Taylor and Francis, 1990).

[15] Н.А. Поклонский, С.А. Вырко, А.Г. Забродский. ФТТ, 46 (6), 1071 (2004).

[16] В.Л. Бонч-Бруевич, С.Г. Калашников. Физика полупроводников (М., Наука, 1990) гл. 19.

[17] Н.А. Поклонский, С.А. Вырко. ЖПС, 69 (3), 375 (2002).

[18] N.A. Poklonski, S.A. Vyrko, V.I. Yatskevich, A.A. Kocherzhenko. J. Appl. Phys., 93 (12), 9749 (2003).

[19] N.A. Poklonski, A.A. Kocherzhenko, S.A. Vyrko, A.T. Vlassov. Phys. Status Solidi B, 244 (10) 3703 (2007).

[20] N.L. Lavrik, V.P. Voloshin. J. Chem. Phys., 114 (21), 9489 (2001).

[21] J. Robertson. Phil. Mag. B, 66 (2), 199 (1992).

[22] B.M. Askerov. Electron Transport Phenomena in Semiconductors (Singapore, World Scientific, 1994).

[23] B.I. Shklovskii, A.L. Efros. Electronic Properties of Doped Semiconductors (Berlin, Springer, 1984).

[24] E.O. Kane. Solid-State Electron., 28 (1, 2), 3 (1985).

[25] Н.А. Поклонский, С.А. Вырко, О.Н. Поклонская, А.Г. Забродский. ФТП, 50 (6), 738 (2016).

[26] N.A. Poklonski, S.A. Vyrko, O.N. Poklonskaya, A.I. Kovalev, A.G. Zabrodskii. J. Appl. Phys., 119 (24), 245701 (2016).

[27] Quantum and Semi-classical Percolation and Breakdown in Disordered Solids, ed. by A.K. Sen, K.K. Bardhan and B.K. Chakrabarti (Berlin, Springer, 2009).

[28] Дж. Займан. Модели беспорядка. Теоретическая физика однородно неупорядоченных систем (М., Мир, 1982) гл. 13.

[29] Н.А. Поклонский, С.А. Вырко, С.Л. Поденок. Статистическая физика полупроводников (М., КомКнига, 2005).

[30] А.И. Ансельм. Введение в теорию полупроводников (М., Наука, 1978) гл. 8.

[31] K. Seeger. Semiconductor Physics: An Introduction (Berlin, Springer, 2004) chap. 6.

[32] V. Palenskis. World J. Condens. Matter Phys., 4 (3), 123 (2014).

[33] E. Conwell, V.F. Weisskopf. Phys. Rev., 77 (3), 388 (1950).

[34] B.K. Ridley. Quantum Processes in Semiconductors (Oxford, Oxford University Press, 2013) chap. 4.

[35] V.G. Baryshevskii, I.D. Feranchuk, P.B. Kats. Phys. Rev. A, 70, 052701 (2004).

[36] C. Hamaguchi. Basic Semiconductor Physics (Berlin, Springer, 2010) chap. 6.

[37] А.Я. Шик. ФТП, 17 (12), 2220 (1983).

[38] В.А. Гергель, Р.А. Сурис. ФТП, 12 (10), 2055 (1978).

[39] D. Chattopadhyay, H.J. Queisser. Rev. Mod. Phys., 53 (4), 745 (1981).

[40] M.J. Katz. Phys. Rev., 140 (4A), 1323 (1965).
[41] H. Fritzsche. J. Phys. Chem. Sol., 6 (1), 69 (1958).

[42] Y. Furukawa. J. Phys. Soc. Jpn., 17 (4), 630 (1962).

[43] J.R. Meyer, F.J. Bartoli. Phys. Rev. B, 36 (11), 5989 (1987).

[44] J.B. Krieger, T. Meeks. Phys. Rev. B, 8 (6), 2780 (1973).

[45] П.И. Баранский, В.В. Коломоец, Ю.А. Охрименко. ФТП, 19 (10), 1768 (1985).

[46] J.B. Krieger, J. Gruenebaum, T. Meeks. Phys. Rev. B, 9 (8), 3627 (1974).

[47] Н.А. Якушева, Г.М. Белобородов. Неорг. матер., 26 (1), 9 (1990).

[48] E. Kuphal, A. Schlachetzki, A. Pöcker. Appl. Phys., 17 (1), 63 (1978).

[49] T. Slupinski, M. Molas, J. Papierska. Acta Phys. Polon. A, 116 (5), 979 (2009).

[50] G.B. Stringfellow. J. Appl. Phys., 50 (6), 4178 (1979).

[51] D. Lancefield, A.R. Adams, M.A. Fisher. J. Appl. Phys., 62 (6), 2342 (1987).

[52] R.T. Bate, R.D. Baxter, F.J. Reid, A.C. Beer. J. Phys. Chem. Sol., 26 (8), 1205 (1965).

[53] В.И. Петровский, Н.Н. Соловьев, Э.М. Омельяновский, В.С. Ивлева. ФТП, 12 (10), 1904 (1978).

[54] В.С. Ивлева, М.Н. Кеворков, Р.С. Митрофанова, А.Н. Попков, В.И. Селянина. ФТП, 12 (3), 534 (1978).

[55] E. Litwin-Staszewska, W. Szymańska, R. Piotrzkowski. Phys. Status Solidi B, 106 (2), 551 (1981).

[56] O. Madelung. Semiconductors: Data Handbook (Berlin, Springer, 2004).

[57] S. Adachi. Properties of Semiconductor Alloys: Group-IV, III-V and II-VI Semiconductors (Chippenham, Wiley, 2009).

[58] В.Т. Бублик, С.С. Горелик, А.Н. Дубровина. ФТТ, 10 (9), 2846 (1968).

[59] В.В. Емцев, М.И. Клингер, Т.В. Машовец. Письма ЖЭТФ, 19 (9), 575 (1974).

[60] R.S. Sorbello. Phys. Status Solidi B, 100 (1), 347 (1980).

[61] М. Шур. Современные приборы на основе арсенида галлия (М., Мир, 1991).

[62] R.P. Joshi, D.K. Ferry. Semicond. Sci. Technol., 7 (3B), B319 (1992).

[63] H. Brooks. Phys. Rev., 83 (4), 879 (1951).

[64] Л.П. Кудрин. Статистическая физика плазмы (М., Атомиздат, 1974) гл. 1, 2.

[65] Д.А. Франк-Каменецкий. Лекции по физике плазмы (М., Атомиздат, 1968) гл. 1.

[66] A. Schmid. Z. Physik, 271 (3), 251 (1974).

[67] Y.J. Zhang, Z.Q. Li, J.J. Lin. EPL, 103, 47002 (2013).

[68] А.В. Дмитриев. ФТТ, 32 (12), 3647 (1990).

[69] В.И. Окулов. Физика низких температур, 30 (11), 1194 (2004).

[70] Б.М. Смирнов. УФН, 138 (3), 517 (1982).

Редактор Г.А. Оганесян 


\section{Quasi-classical model of static electrical conductivity of heavily doped degenerate semiconductors at low temperatures}

N.A. Poklonski, S.A. Vyrko, A.N. Dzeraviaha

Belarusian State University, 220030 Minsk, Belarus

Abstract The crystals of germanium, silicon, gallium arsenide and indium antimonide of $n$-type are considered on the metallic side of the insulator-metal transition (Mott transition). The static (direct current) electrical conductivity and the drift mobility of $c$-band electrons as well as the electrostatic fluctuations of their potential energy and the mobility edge are calculated in quasiclassical approximation. It is considered that a single act of elastic Coulomb scattering of a mobile electron takes place only in a spherical region of crystal matrix in the center of which the impurity ion is located. The results of calculations using proposed formulas without using any adjustable parameters numerically agree with the experimental data in a wide range of hydrogenlike donor concentration at weak and moderate compensation by acceptors. 